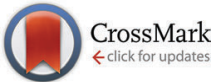

Cite this: J. Mater. Chem. B, 2016, 4, 5228

Received 15th April 2016,

Accepted 5th July 2016

DOI: $10.1039 / c 6 t b 00942 e$

www.rsc.org/MaterialsB

\section{Vertically aligned multi walled carbon nanotubes prevent biofilm formation of medically relevant bacteria†}

\begin{abstract}
I. Malek, ${ }^{a}$ C. F. Schaber, ${ }^{b}$ T. Heinlein, ${ }^{c}$ J. J. Schneider, ${ }^{c}$ S. N. Gorb ${ }^{b}$ and R. A. Schmitz ${ }^{a}$
A significant part of human infections is frequently associated with the establishment of biofilms by (opportunistic) pathogens. Due to the increasing number of untreatable biofilms, there is a rising need to develop novel and effective strategies to prevent biofilm formation on surfaces in medical as well as in technical areas. Bacterial initial attachment and adhesion to surfaces followed by biofilm formation is highly influenced by the physical properties of the surfaces. Consequently, changing these properties or applying different nanostructures is an attractive approach to prevent biofilm formation. Here we report on the effect(s) of surface grown and anchored vertically aligned multi walled carbon nanotubes (MWCNT), which have been made wettable by immersion through a graded ethanol series, on biofilm formation of Klebsiella oxytoca, Pseudomonas aeruginosa, and Staphylococcus epidermidis. We evaluated the biofilm formation under continuous flow conditions by confocal laser scanning microscopy and scanning electron microscopy, and demonstrated significant inhibition of biofilm formation of all the different pathogens by MWCNT of different lengths. Furthermore, the anti-adhesive effects of the MWCNT increased with their overall length. The application potential of our findings on surface grown and anchored vertically aligned MWCNT may represent a suitable contact mechanics based approach to prevent biofilm formation on medical devices or technical sensors operating in fluid environments.
\end{abstract}

\section{Introduction}

Bacteria and archaea are able to adhere to abiotic as well as biotic surfaces by means of their adhesins, cell-surface components or appendages such as pili, fimbriae, flagella and slime; subsequently establishing biofilms. ${ }^{1,2}$ These biofilms are highly dynamic microbial communities adhered to a surface consisting of one or more species often embedded in an extracellular matrix., In general, the ability to develop biofilms on surfaces or interfaces represents a predominant strategy of microorganisms to survive conditions of nutrient-limitation. ${ }^{5}$

Besides numerous beneficial biofilms e.g. biofilms in the environment degrading toxic compounds ${ }^{6,7}$ or application of biofilms in the treatment of wastewater, ${ }^{8,9}$ bacteria are also able to form harmful biofilms e.g. those on technical equipment as well as on medical devices and implants. Prominent examples

\footnotetext{
${ }^{a}$ University of Kiel, Institute for General Microbiology, Kiel 24118, Germany. E-mail: rschmitz@ifam.uni-kiel.de

${ }^{b}$ University of Kiel, Zoological Institute, Functional Morphology and Biomechanics, Kiel 24118, Germany

${ }^{c}$ Technische Universität Darmstadt, Fachbereich Chemie, Eduard-Zintl-Institut für Anorganische und Physikalische Chemie, Darmstadt 64287, Germany

$\dagger$ Electronic supplementary information (ESI) available: $P$-values (Statistical analysis) and live/dead ratios. See DOI: 10.1039/c6tb00942e
}

are pathogenic bacteria such as Staphylococcus epidermidis and Pseudomonas aeruginosa, which are frequently linked to biofilm-associated infections. ${ }^{10-12}$ These harmful biofilms represent a major challenge because the approaches to combat them are limited. Currently, chemical compounds such as disinfectants and antibiotics are mainly used to inhibit medicallyrelevant biofilm formation. However, once organized in a biofilm, microorganisms show significantly higher resistance to disinfectants, ${ }^{13}$ antibiotics ${ }^{14}$ and host's defense by the (innate) immune system. ${ }^{15}$ Consequently, there is a lack of effective substances and general strategies to inhibit biofilm formation of disruptive or pathogenic bacteria on medical surfaces and novel approaches are urgently required.

Biofilm formation generally comprises four developmental steps-initial attachment, irreversible attachment, maturation, and dispersion. ${ }^{16}$ The initial phase also called initiation or adhesion phase is influenced by numerous factors including physico-chemical properties of the surface, environmental conditions and molecular interactions ${ }^{17}$ as well as bacterial motility. ${ }^{3}$ Due to the fact that initial adhesion of microorganisms is crucially influenced by the properties of the respective surface, changing chemical and physical properties of a surface provide an encouraging strategy to inhibit biofilm formation. Consequently, applying different nanostructures to the surface is a promising 
approach to prevent adhesion and subsequent biofilm formation on medical and technological devices. In this respect, carbon nanotubes (CNT) are attractive nanostructures for such an approach. CNT are allotropes of elemental carbon with a cylindrical nanostructure forming well-ordered tubes and can be categorized in single-walled carbon nanotubes (SWCNT), double walled carbon nanotubes (DWCNT) and multi-walled carbon nanotubes (MWCNT) ${ }^{18}$ Until today, analysing nanotubes with regard to inhibitory effects on microorganisms mainly focused on evaluating the effectiveness of randomly oriented CNT on planktonically growing microorganisms. ${ }^{19-27}$ Only one study investigated the effects of (non-aligned) randomly oriented CNT on biofilm formation of Escherichia coli growing as small batch cultures in 96-well microtiter plates. Here it was demonstrated that using this system the presence of SWCNT was efficient to reduce biofilm formation. ${ }^{28}$

To the best of our knowledge only one recent report evaluated the effects of vertically aligned $\mathrm{CNT}^{29}$ In that study, bacterial adhesion on MWCNT treated with argon at $5 \mathrm{~Pa}$ (plasma treatment) was evaluated during growth in batch cultures (24-well microtiter plates). In contrast, the present work aimed to study the effects of wettable vertically aligned MWCNT of two different lengths on biofilm formation of various human opportunistic pathogens using flow chambers with a continuous flow; a recent setting representing conditions close to the natural environmental situation e.g. in pipelines, tubes, and venous catheters.

\section{Experimental section}

\subsection{Bacterial strains and growth conditions}

The bacterial strains Klebsiella oxytoca M5a $1,^{30}$ Pseudomonas aeruginosa $\mathrm{PAO} 1,^{31}$ Staphylococcus epidermidis $1457^{32}$ and K. pneumoniae spec. [kindly provided by Prof. Podschun (ESBL No. 81, reference laboratory CAU Kiel)] were used as model organisms for biofilm formation. Batch cultures of Klebsiella oxytoca M5a 1 and $K$. pneumoniae spec. were grown over night in LB (Luria-Bertani) medium ${ }^{33}$ at $30^{\circ} \mathrm{C}$. Pseudomonas aeruginosa PAO1 and Staphylococcus epidermidis 1457 were also grown over night in LB medium but at their optimum temperature of $37{ }^{\circ} \mathrm{C}$.

In order to evaluate biofilm formation, strains were grown in flow chambers (see below) using strain specific media. The $P$. aeruginosa and $S$. epidermidis biofilms were cultured in highly diluted (1:33) Tryptonic Soya Broth (TSB). ${ }^{34}$ In the case of the Klebsiella strains, GC minimal medium ${ }^{35}(1 \%$ (v/v) glycerol and $0.3 \%(\mathrm{w} / \mathrm{v})$ casamino acids) was used.

\subsection{Growth parameters of carbon nanotubes}

Vertically aligned MWCNT were grown by chemical vapor deposition (CVD). First, $11.6 \mathrm{~nm} \mathrm{Al} \mathrm{metal} \mathrm{followed} \mathrm{by} 1.4 \mathrm{~nm}$ Fe metal were deposited by e-beam evaporation on a silicon substrate (boron doped $\langle 100\rangle$ coated with a $600 \mathrm{~nm}$ layer of silicon dioxide). After heating the substrate up to $850{ }^{\circ} \mathrm{C}$ in a gas mixture of $\mathrm{Ar} / \mathrm{H}_{2}\left(40 \% \mathrm{H}_{2}\right.$, total flow $\left.1400 \mathrm{sscm}\right)$ in a tubular furnace with a quartz tube (inner diameter $85 \mathrm{~mm}$ ), CVD synthesis was initiated by introducing $200 \mathrm{sscm}$ ethylene and $420 \mathrm{ppm}$ of water vapor. Growth and alignment of CNT of two different lengths ( 470 and $540 \mu \mathrm{m})$ were controlled by variation of the growth time. For further details of the growth process and properties of the MWCNT arrays see. ${ }^{36-38}$

\subsection{Biofilm formation in flow chambers}

Coverslips without MWCNT, as control, and coverslips with the MWCNT samples were inserted into the flow chambers and sealed with polyvinylsiloxane (President Light Body, Coltène/ Whaledent AG, Altstätten Switzerland). The complete chambers (total volume $1.3 \mathrm{ml}$ ) were attached to a peristaltic pump (Ismatec ISM931C, Germany) and equilibrated with the respective medium for $2 \mathrm{~h}$ with a flow rate of $20 \mathrm{ml} \mathrm{h}^{-1}$ or $30 \mathrm{ml} \mathrm{h}^{-1}$ depending on the microorganism (see below). The biofilm assays of $K$. oxytoca M5al and $K$. pneumoniae isolate 81 were performed in GC minimal medium (flow rate $20 \mathrm{ml} \mathrm{h}^{-1}$ ) at $30{ }^{\circ}$ C. $P$. aeruginosa PAO1 and $S$. epidermidis 1457 biofilms were grown in highly diluted $(1: 33)$ TSB (flow rate of $30 \mathrm{ml} \mathrm{h}^{-1}$ ) at $37{ }^{\circ} \mathrm{C}$. Overnight cultures were adjusted to $1 \times 10^{8}$ cells per $\mathrm{ml}$ for Klebsiella strains and to $3 \times 10^{8}$ cells per $\mathrm{ml}$ for $S$. epidermidis and $P$. aeruginosa. After supplementing the flow chamber with $1.3 \mathrm{ml}$ adjusted cultures and further incubation for $1 \mathrm{~h}$ without medium flow, the $K$. pneumoniae isolate 81 , $P$. aeruginosa PAO1 and $S$. epidermidis 1457 were cultivated for $20 \mathrm{~h}$ under continuous flow conditions, whereas $K$. oxytoca M5al was grown in flow chambers for $72 \mathrm{~h}$. Subsequently all samples were stained, visualized and microscopically analysed (see below).

\subsection{Preparation of CNT samples for flow chamber assay}

The samples of MWCNT of different length firmly attached to the silicon substrate were fixed on coverslips (Menzel Glaeser, Braunschweig, Germany) using polyvinylsiloxane (President Light Body, Coltène/Whaledent AG, Altstätten Switzerland) and inserted into the flow chambers. The samples were made wettable prior the biofilm assays with ethanol of 100, 70, 50, 30 and $10 \%$ (vol/vol); each concentration was used for $20 \mathrm{~min}$ with a flow of $20 \mathrm{ml} \mathrm{h}^{-1}$ or $30 \mathrm{ml} \mathrm{h}^{-1}$ and equilibrated with the respective medium for $2 \mathrm{~h}$ with a flow rate of $20 \mathrm{ml} \mathrm{h}^{-1}$ or $30 \mathrm{ml} \mathrm{h}^{-1}$ depending on the microorganism (see above).

\subsection{Microscopy}

The biofilms for confocal laser scanning microscopy (CLSM) were stained with the FilmTracerTM LIVE/DEAD ${ }^{\circledR}$ Biofilm Viability Kit (Life Technologies, Darmstadt, Germany) according to the manufacturer's protocol and visualized using a LSM 700 microscope (Zeiss; Jena, Germany). Maximum biofilm thickness was determined from the CLSM images by manually evaluating the various levels within the sample. The biofilm volume was determined by ImageJ. ${ }^{39}$ Briefly, the fluorescence intensity of each pixel was determined using the plugin $3 \mathrm{D}$ object counter of Image J and integrated. 3D surface plots of the biofilm were generated using the interactive 3D surface plot plugin 2.32 of ImageJ.

For scanning electron microscopy (SEM), the samples were fixed in $2.5 \%$ glutaraldehyde overnight at $4{ }^{\circ} \mathrm{C}$. After fixation, the samples were dehydrated in 15, 25, 40, 55, 70, 85, 95 and $100 \%$ ethanol each for $20 \mathrm{~min}^{40}$ and critical point dried 
(Polaron CPD E3000 combined with Heater/chiller E4860). Afterwards the samples were sputter-coated with $5 \mathrm{~nm}$ gold/palladium and the images captured using SEM S-4800 (Hitachi).

\subsection{Statistical analysis}

Unpaired $t$-test was performed comparing thickness of the biofilms developed on glass controls with those on the MWCNT. Biofilm volume was compared respectively. Statistics were performed with GraphPad Prism 6 software (GraphPad, San Diego, CA, USA) with differences ${ }^{*} P<0.05$ considered significant. The respective $P$-values are summarized in the Tables S2 and S3 (ESI $\dagger$ ).

\section{Results and discussion}

\subsection{Bacterial biofilm formation on MWCNT}

Vertically aligned multi walled carbon nanotubes MWCNT (see Fig. 1) of two different lengths (470 and $540 \mu \mathrm{m})$ were grown by a catalyst driven CVD process on silicon wafers (see Experimental section). Different growth heights were obtained by varying the growth time. ${ }^{36}$ The MWCNT within the arrays are double to six-walled and their diameter is in the range between $6-20 \mathrm{~nm} \cdot{ }^{37}$ The respective inter tube distance is between 10 and $20 \mathrm{~nm}$ maximally. ${ }^{41,42}$ The growth on the silicon surface led to a secure anchoring of the CNT and assured that they were not detached and washed out of the flow chamber during the entire experiment. This finding is especially relevant since shear forces caused by surface drag might be rather strong under such conditions. The obtained MWCNT have been made wettable (hydrophilic) as described in the Experimental section.

At first, biofilm formation of three opportunistic human pathogens - S. epidermidis, $P$. aeruginosa and $K$. oxytoca - was evaluated on hydrophilic $470 \mu \mathrm{m}$ long MWCNT in flow chambers under a continuous constant flow of the respective medium (see Experimental section). Biofilms established after $20 \mathrm{~h}$ in case of the coccus $S$. epidermidis (diameter $0.5-1.0 \mu \mathrm{m}$ ) and the rod-shaped $P$. aeruginosa $(2-3 \mu \mathrm{m}$ length and $0.5-0.7 \mu \mathrm{m}$ width) and $72 \mathrm{~h}$ in case of the rod-shaped $K$. oxytoca $(2.5 \mu \mathrm{m}$ length and $0.5-0.7 \mu \mathrm{m}$ width) were visualized and compared to control biofilms established on the control surface using two microscopic methods: Confocal laser scanning microscopy (CLSM) and scanning electron microscopy (SEM). Evaluating various biofilm characteristics, we discovered that the volume represents an adequate read-out and an important characteristic of biofilms allowing the comparison of biofilm formation and the evaluation of inhibitory effects on biofilm formation. Thus, besides the thickness of the respective biofilms, analysed by a surface plot, the volumes of the biofilms were calculated based on the respective 3D CLSM data sets (see Experimental procedures).

The established biofilms on MWCNT $(470 \mu \mathrm{m})$ showed significant differences compared to the controls in all cases (see Fig. 2). Biofilm formation of $S$. epidermidis was highly affected by MWCNT, resulting in a significant volume reduction of down to $8 \%$ in comparison to control on glass (Fig. $2 \mathrm{c}$ and d). $K$. oxytoca biofilms were significantly reduced down to $26 \pm 10 \%$ of the control volume (Fig. 2a and d), whereas in case of $P$. aeruginosa only partial inhibition of biofilm formation was obtained, reducing the volume down to $60 \pm 15 \%$ (Fig. $2 \mathrm{~b}$ and d). Moreover, in contrast to the compact mature biofilms established on glass, biofilms formed on the MWCNT by $P$. aeruginosa and $K$. oxytoca appeared structurally loose; whereas $S$. epidermidis developed only individual small micro-colonies (see Fig. 2a-c CLSM images, middle panel). SEM analysis after fixation of established biofilms with glutaraldehyde confirmed the changes and strong reduction of $S$. epidermidis and $K$. oxytoca biofilm formation on the MWCNT,
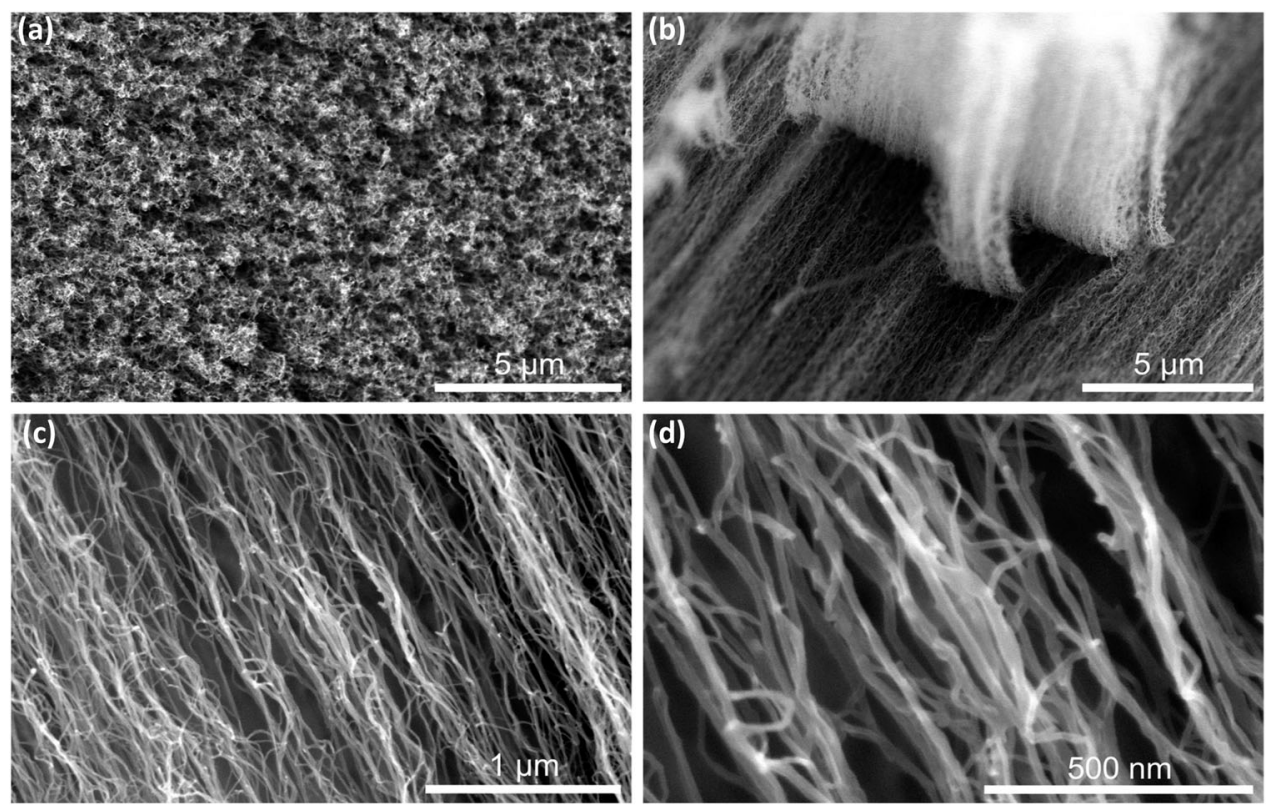

Fig. 1 Vertically aligned MWCNT visualized with SEM. Top view (a) and side views at different magnifications (b-d) of the MWCNT 

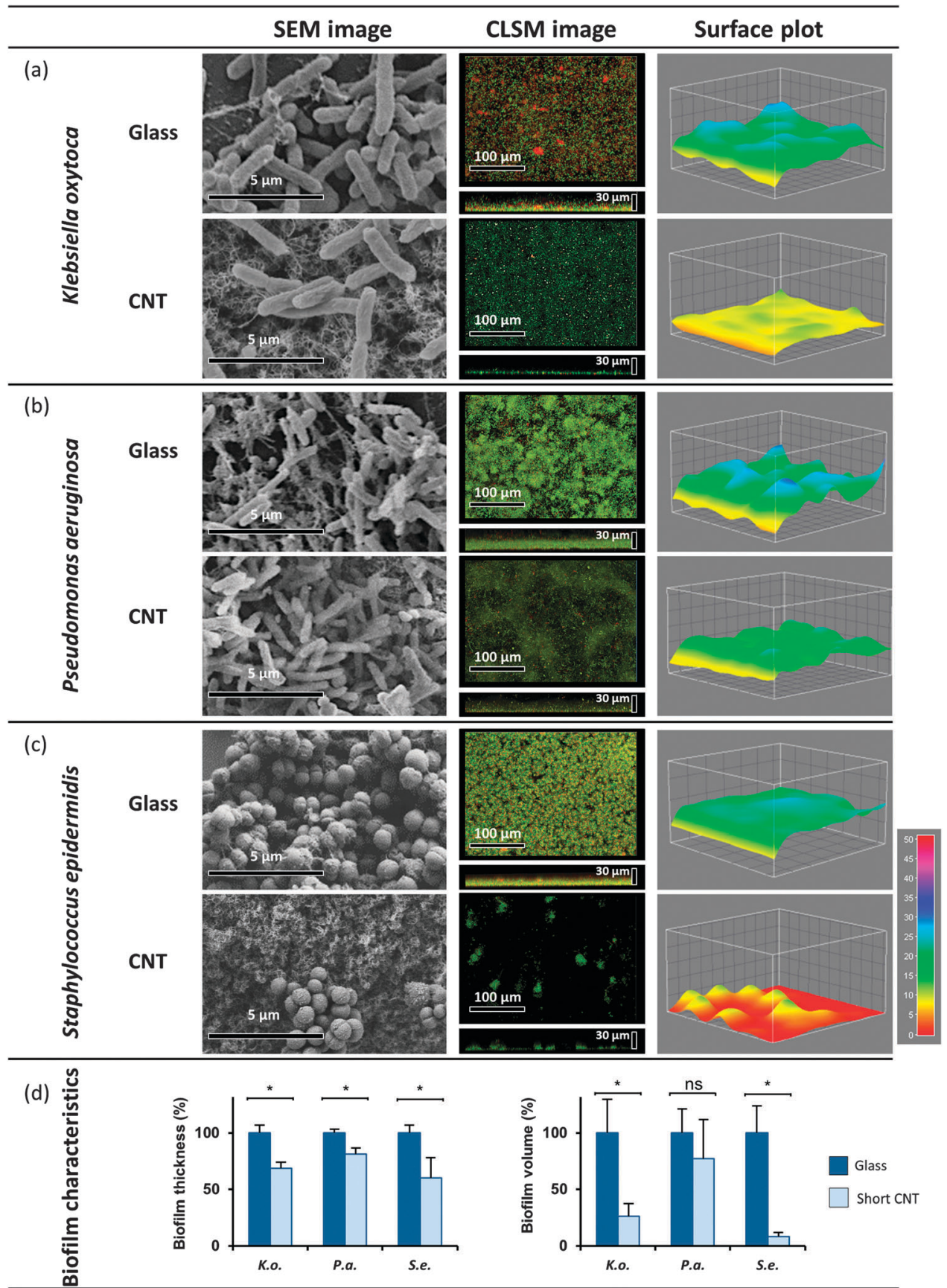

Fig. 2 Biofilm formation on MWCNT. Established biofilms of $K$. oxytoca (a), P. aeruginosa (b) and S. epidermidis (c) on MWCNT (470 $\mu$ m), and on glass as a control were analyzed with SEM and CLSM (see Experimental section). (d) The biofilm characteristics thickness and volume were quantified with ImageJ. Means of at least four independent biological replicates are indicated.

while $P$. aeruginosa biofilm formation appeared to be only marginally influenced (Fig. 2a-c, SEM images, left panel).

\subsection{Characterization of biofilm formation on longer MWCNT}

In a second similar experimental set-up, biofilm formation of the three opportunistic human pathogens was evaluated on $540 \mu \mathrm{m}$ long MWCNT. Evaluating the biofilm volume demonstrated that in contrast to the shorter MWCNT, the biofilm formation of all tested model organisms was highly affected on $540 \mu \mathrm{m}$ long
MWCNT. CLSM analysis revealed significant reduction of $K$. oxytoca and $S$. epidermidis biofilm volumes down to $2 \%$, and reduction of $P$. aeruginosa biofilm volume down to $13 \%$ (see Fig. 3d). The drastic change in the overall structure of the biofilms was confirmed by SEM (Fig. 3a-c, left panel).

\subsection{Clinical isolate: reduced biofilm volume on MWCNT}

The selected and evaluated strains of $K$. oxytoca, $P$. aeruginosa and $S$. epidermidis represent type strains cultivated for a high 


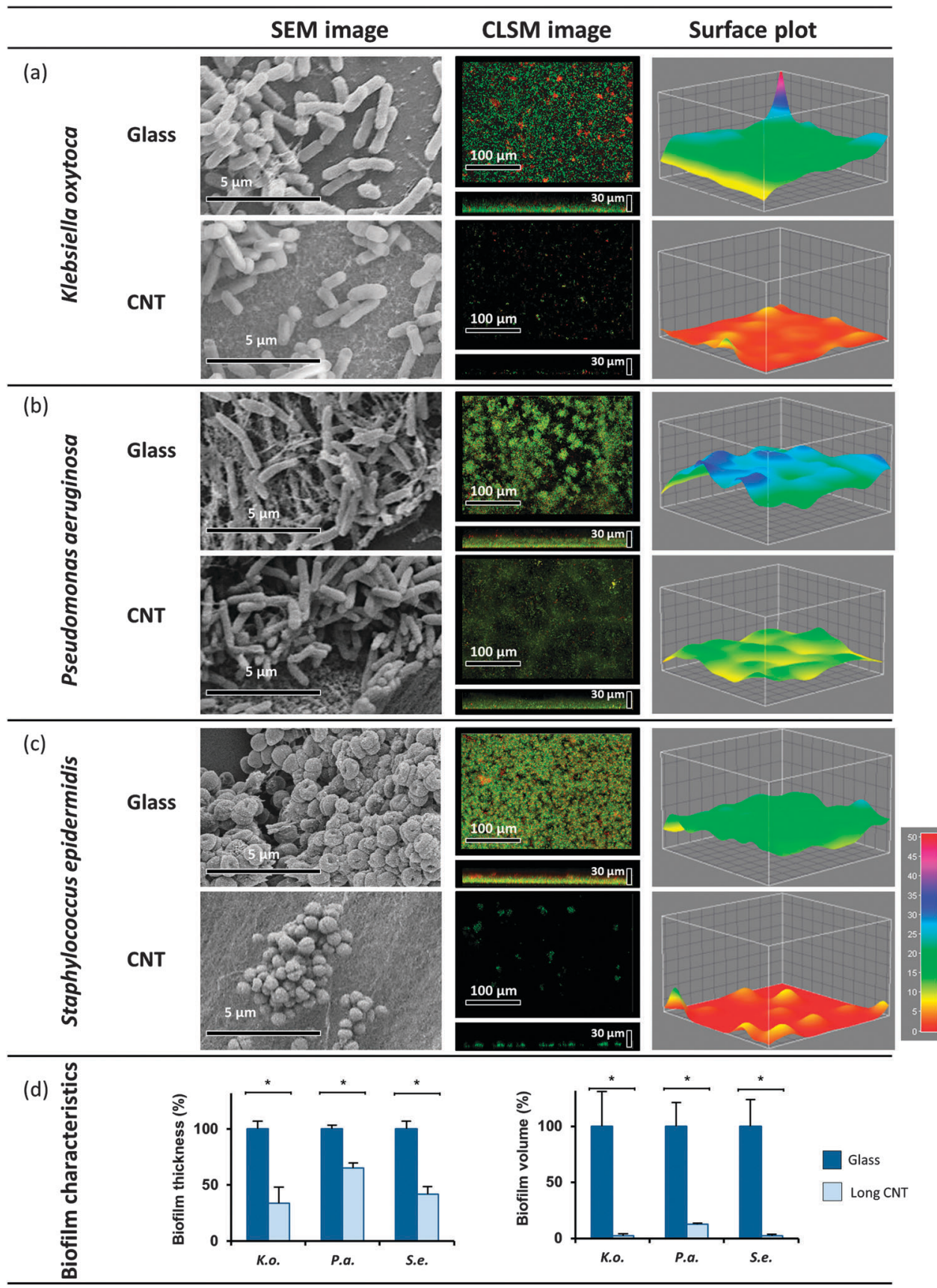

Fig. 3 Biofilm formation on longer MWCNT. Established biofilms of K. oxytoca (a), P. aeruginosa (b) and S. epidermidis (c) on longer MWCNT (540 $\mu$ m) were analyzed. A control on glass was performed for all model organisms. The visualization was carried out with SEM and CLSM. The biofilm characteristics (d) were quantified with ImageJ. Means of at least three biological replicates are indicated.

number of generations under laboratory conditions, and thus might not completely reflect the physiology and biofilm formation of pathogenic strains associated with the human host. Consequently, a clinical isolate of $K$. pneumoniae was additionally included in the present study, K. pneumoniae spec. isolated from a urinary catheter (ESBL No. 81, reference laboratory CAU Kiel, Prof. Dr Podschun). The biofilm formation of this K. pneumoniae isolate on vertically aligned MWCNT $(470 \mu \mathrm{m})$ in the flow chamber demonstrated significant reduction of the biofilm volume (down to $32 \%$ ) compared to the control (Fig. 4).
This finding is in agreement and in the same range of the volume reduction obtained for the type strain $K$. oxytoca, and thus proves that the biofilm formation of the clinical isolate is equally influenced by MWCNT of $470 \mu \mathrm{m}$ length.

Overall, these findings indicate a strong impact of the vertically aligned MWCNT on biofilm formation of $K$. oxytoca, $P$. aeruginosa, and $S$. epidermidis. Remarkably, Pantanella et $a l^{43}$ demonstrated that coating surfaces by single-walled CNT is apparently not suitable to inhibit biofilm formation of Streptococcus mutans and P. aeruginosa. The absence of inhibitory 

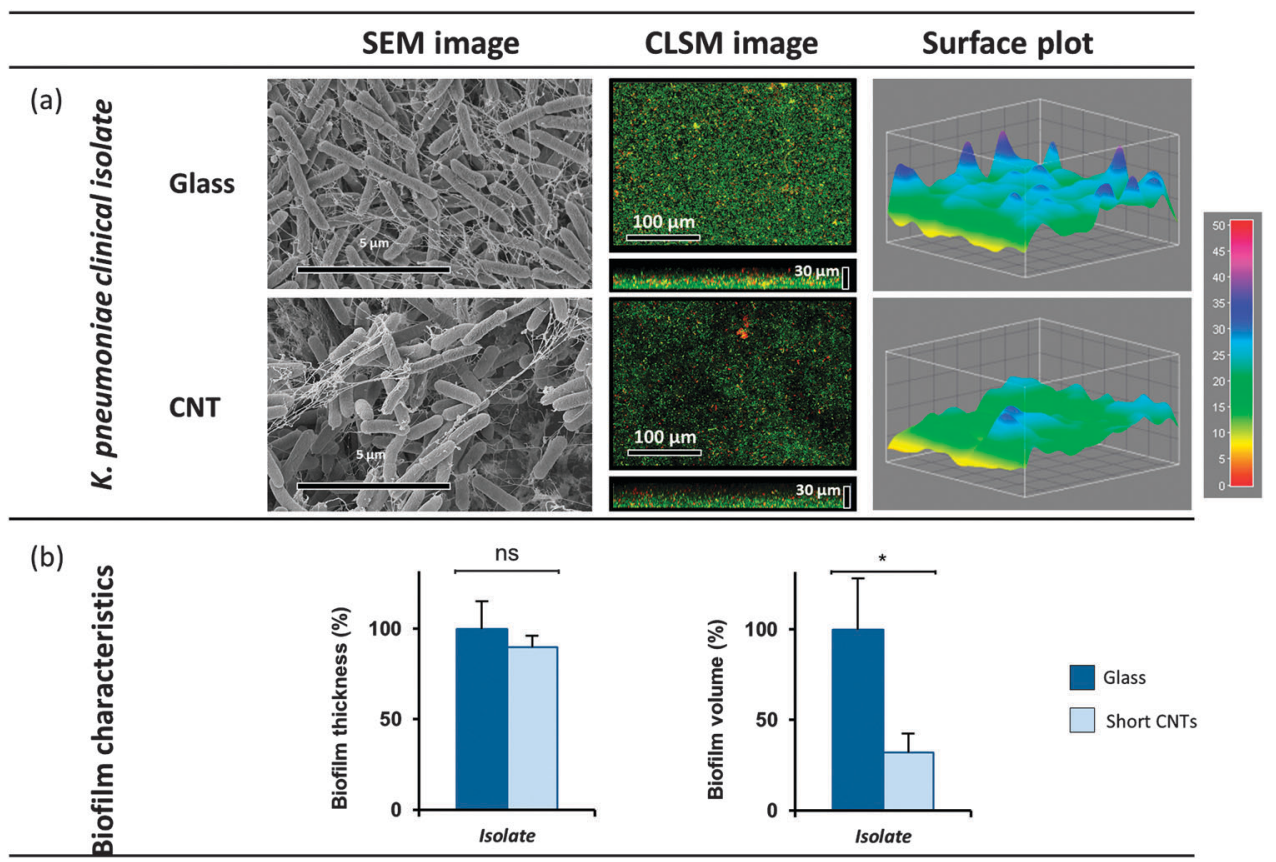

Fig. 4 (a) Biofilm formation of a K. pneumoniae clinical isolate on MWCNT. The biofilm analyses of the MWCNT (470 $\mu \mathrm{m})$, were carried out with SEM and CLSM. (b) Thickness and volume of the biofilms were quantified with ImageJ. Means of at least three independent biological replicates are indicated.

effects in that study may be explained by the fact that MWCNT were firmly attached to the surface by their walls and therefore presumably formed a rigid surface exposed to the environment. Also Yick et al. ${ }^{29}$ who studied the effects of immobilized MWCNT in 24 well plastic plates observed no effects on cell counts and viability of $P$. aeruginosa due to the MWCNT presence. However, unfortunately no direct evaluation of biofilm formation was performed, despite the fact that the experimental set up in our study differed considerably (flow conditions, wettability of MWCNT).

\subsection{Inhibition of biofilm formation by nanotubes: possible mechanisms}

Currently, the effectiveness of CNT on microorganisms is controversially discussed. Several reports described a strong effect of CNT on bacterial planktonic cells. For instance, Rajavel et al. demonstrated strong negative effects of randomly oriented CNT on the growth of four bacterial species including $K$. pneumoniae and $P$. aeruginosa. ${ }^{23,28}$ They observed a strong relation to reactive oxidative species (ROS) production by the CNT. Besides, partial penetration of the nanotubes into the cells $^{22}$ is speculated and discussed as a potential mechanism of inhibition. ${ }^{24}$ Yick et al. ${ }^{29}$ also detected weak generation of ROS during their study and assumed penetration into the cells as the cause for reduced bacterial adhesion. Finally, Zhu et al. ${ }^{25}$ recently reported that bacteria are able to compensate for those CNT effects by increasing the membrane fluidity. However, the overall mechanism for inhibition of planktonic cells is still not clarified and highly debated.

In contrast to all previous studies, the present report aimed for the first time to evaluate the impact of MWCNT of defined and different lengths on bacterial biofilm formation under flow condition. In addition, instead of using randomly oriented CNT, which have been shown to effect planktonic cells ${ }^{19-25,44}$ MWCNT were directly synthesized and anchored on a silicon surface leading to immobilized vertically aligned MWCNT arrays. Analysing the ratio between living and dead cells in established biofilms by CLSM analysis after staining with syto9 and propidium iodide revealed a similar ratio under both conditions - control surface (glass) and immobilized MWCNT or even higher relative amounts of living cells on the MWCNT (see Table S1, ESI $\dagger$ ). This finding strongly argues against toxic effects of anchored MWCNT on the cells by e.g. ROS or CNT penetration into the cells. The latter was further verified by SEM images, revealing that cells adhered to the MWCNT were not penetrated by the MWCNT. Those findings are in agreement with previous studies using cortical neurons which showed that neither the vertically aligned dense carbon nanotube networks nor the respective biomaterials used during the generation of the MWCNT showed toxic effects on neurons. ${ }^{45}$ Consequently, we propose that toxic effects of MWCNT can be excluded as a reason for the strong inhibition of biofilm formation observed in our study.

On the other hand, the microtexture is discussed as a reason for observed negative effects of nanostructures on cell adhesion. Ma et al. $^{46}$ incubated various fabricated fibrillar polypropylene (PP) nanoarrays with two different algae species and detected low cell adhesion when the interval of the PP fibers was smaller than the cell size. While higher adhesion was detected, when the interval of the fibers was larger than the cells. These results are in agreement with the attachment point theory, which was proposed as a mechanism for biofouling on microtextured surfaces. ${ }^{47}$ Since the microtexture of shorter and longer MWCNT 
used in this study is identical, the obtained different effects on biofilm formation cannot be explained by the attachment point theory.

Consequently, we propose that the anti-adhesive effect is rather based on the mobility of MWCNT leading to an unstable substrate affecting proper contact formation by bacterial cells. The strong inhibitory effects on the biofilm formation drastically increased with the length of the vertically aligned MWCNT. These different effects due to size is in agreement with findings studying the effects of non-immobilized nanotubes on bacterial growth, which showed that the size of free nanotubes appears to play a role for effecting planktonic bacteria (targeting growth and viability). ${ }^{22,24}$ Shorter vertically aligned MWCNT are more rigid and only slightly move, if at all, due to the medium flow. The longer MWCNT are more flexible and might oscillate preventing bacterial settlement. Thus, the second discovery indicates that besides strong anti-adhesive effects of MWCNT, the potentially increased flexibility of longer MWCNT are not providing a static surface for the bacterial adhesins and is additionally affecting biofilm formation.

\section{Conclusions}

In summary, we evaluated $K$. pneumoniae, $K$. oxytoca, $P$. aeruginosa and $S$. epidermidis biofilm formation on surface anchored vertically aligned MWCNT of two different lengths using flow chambers with continuous flow. This is the first study demonstrating the strong impact of immobilized MWCNT on biofilm formation of selected human pathogens in flow chambers; moreover this inhibition considerably increased with the length of MWCNT. Based on our findings, we propose that the impact of long but still firmly attached CNT arranged in a homogeneously ordered microscopic array of aligned MWCNT might have a substantial impact for future medical applications. They might be able to avoid e.g. infections caused by biofilms or contaminations of surfaces of technologically relevant devices, such as flow sensors in a fluid environment rich on bacteria due to their herein proven antibacterial behaviour.

\section{Acknowledgements}

Irene Malek was funded by the Excellence cluster 'The Future Ocean'. We gratefully acknowledge Prof. Podschun (University Medical Center Schleswig-Holstein, UKSH, Kiel, Germany) for kindly providing the clinical Klebsiella pneumoniae isolate and Christopher Borowski for performing the flow chamber experiments with Klebsiella pneumoniae isolate. We thank Dr AlexandraSophie Roy (Microbiology, University of Kiel) for the linguistic revision of the manuscript. Thorsten Heinlein and Jörg J. Schneider gratefully acknowledge support through the LOEWE program Sensors towards Terahertz (STT) funded by the state of Hessen.

\section{References}

1 G. E. Soto and S. J. Hultgren, J. Bacteriol., 1999, 181, 1059-1071.

2 C. Bang, C. Ehlers, A. Orell, D. Prasse, M. Spinner, S. N. Gorb, S. V. Albers and R. A. Schmitz, Frontiers in Microbiology, 2014, 5, 353.

3 G. A. O’Toole and R. Kolter, Mol. Microbiol., 1998, 30, 295-304.

4 M. E. Davey and G. A. O’Toole, Microbiol. Mol. Biol. Rev., 2000, 64, 847-867.

5 R. Dewanti and A. C. L. Wong, Int. J. Food Microbiol., 1995, 26, 147-164.

6 T. C. Zhang, Y. C. Fu, P. L. Bishop, M. Kupferle, S. Fitzgerald, H. H. Jiang and C. Harmer, J. Hazard. Mater., 1995, 41, 267-285.

7 R. Singh, D. Paul and R. K. Jain, Trends Microbiol., 2006, 14, 389-397.

8 I. Schmidt, O. Sliekers, M. Schmid, E. Bock, J. Fuerst, J. G. Kuenen, M. S. M. Jetten and M. Strous, FEMS Microbiol. Rev., 2003, 27, 481-492.

9 G. E. Petrie and F. S. Lupton, Waste Manage. Res., 1991, 9, 471-476.

10 U. Romling and C. Balsalobre, J. Intern. Med., 2012, 272, 541-561.

11 L. Claret, S. Miquel, N. Vieille, D. A. Ryjenkov, M. Gomelsky and A. Darfeuille-Michaud, J. Biol. Chem., 2007, 282, 33275-33283.

12 Y. Kumagai, J. Matsuo, Z. H. Cheng, Y. Hayakawa and Y. Rikihisa, Infect. Immun., 2011, 79, 3905-3912.

13 L. K. Vestby, T. Moretro, S. Langsrud, E. Heir and L. L. Nesse, BMC Vet. Res., 2009, 5, 20.

14 H. Ceri, M. E. Olson, C. Stremick, R. R. Read, D. Morck and A. Buret, J. Clin. Microbiol., 1999, 37, 1771-1776.

15 G. J. Meluleni, M. Grout, D. J. Evans and G. B. Pier, J. Immunol., 1995, 155, 2029-2038.

16 C. Bordi and S. de Bentzmann, Ann. Intensive Care, 2011, 1, 19.

17 Y. H. An and R. J. Friedman, J. Biomed. Mater. Res., 1998, 43, 338-348.

18 C. Hierold, O. Brand, G. K. Fedder, J. G. Korvink and O. Tabata, Carbon Nanotube Devices: Properties, Modeling, Integration and Applications, Wiley, 2008.

19 A. S. Brady-Estevez, M. H. Schnoor, S. Kang and M. Elimelech, Langmuir, 2010, 26, 19153-19158.

20 S. Kang, M. S. Mauter and M. Elimelech, Environ. Sci. Technol., 2008, 42, 7528-7534.

21 S. Kang, M. Pinault, L. D. Pfefferle and M. Elimelech, Langmuir, 2007, 23, 8670-8673.

22 S. Kang, M. Herzberg, D. F. Rodrigues and M. Elimelech, Langmuir, 2008, 24, 6409-6413.

23 K. Rajavel, R. Gomathi, S. Manian and R. T. R. Kumar, Langmuir, 2014, 30, 592-601.

24 C. N. Yang, J. Mamouni, Y. A. Tang and L. J. Yang, Langmuir, 2010, 26, 16013-16019.

25 B. T. Zhu, X. H. Xia, N. Xia, S. W. Zhang and X. J. Guo, Environ. Sci. Technol., 2014, 48, 4086-4095. 
26 L. R. Arias and L. J. Yang, Langmuir, 2009, 25, 3003-3012.

27 S. B. Liu, L. Wei, L. Hao, N. Fang, M. W. Chang, R. Xu, Y. H. Yang and Y. Chen, ACS Nano, 2009, 3, 3891-3902.

28 D. F. Rodrigues and M. Elimelech, Environ. Sci. Technol., 2010, 44, 4583-4589.

29 S. Yick, A. Mai-Prochnow, I. Levchenko, J. H. Fang, M. K. Bull, M. Bradbury, A. B. Murphy and K. Ostrikov, RSC Adv., 2015, 5, 5142-5148.

30 F. C. Cannon, G. E. Riedel and F. M. Ausubel, Mol. Gen. Genet., 1979, 174, 59-66.

31 R. R. Corwell, Int. Bull. Bacteriol. Nomencl. Taxon., 1965, 15, 87-95.

32 D. Mack, N. Siemssen and R. Laufs, Infect. Immun., 1992, 60, 2048-2057.

33 G. Bertani, J. Bacteriol., 1951, 62, 293-300.

34 J. F. MacFaddin, Media for the isolation - cultivation - maintenance of medical bacteria, Williams and Wilkins, 1985, vol. 1.

35 G. F. Gerlach, B. L. Allen and S. Clegg, J. Bacteriol., 1988, 170, 3547-3553.

36 R. Joshi, J. Engstler, L. Houben, M. Bar Sadan, A. Weidenkaff, P. Mandaliev, A. Issanin and J. J. Schneider, ChemCatChem, 2010, 2, 1069-1073.

37 C. F. Schaber, T. Heinlein, G. Keeley, J. J. Schneider and S. N. Gorb, Carbon, 2015, 94, 396-404.
38 D. J. Babu, S. N. Varanakkottu, A. Eifert, D. de Koning, G. Cherkashinin, S. Hardt and J. J. Schneider, Adv. Mater. Interfaces, 2014, 1, 1300049.

39 W. Burger and M. J. Burge, Digital Image Processing, Springer, London, 2008, ch. 3, pp. 27-36, DOI: 10.1007/ 978-1-84628-968-2_3.

40 P. S. Stewart, R. Murga, R. Srinivasan and D. Debeer, Water Res., 1995, 29, 2006-2009.

41 C. Nick, S. Yadav, R. Joshi, J. J. Schneider and C. Thielemann, Appl. Phys. Lett., 2015, 107, 013101.

42 R. K. Joshi and J. J. Schneider, Chem. Soc. Rev., 2012, 41, 5285-5312.

43 F. Pantanella, F. Berlutti, D. Passeri, D. Sordi, A. Frioni, T. Natalizi, M. L. Terranova, M. Rossi and P. Valenti, Interdiscip. Perspect. Infect. Dis., 2011, 2011, 291513.

44 B. Shrestha, V. Acosta-Martinez, S. B. Cox, M. J. Green, S. Li and J. E. Canas-Carrell, J. Hazard. Mater., 2013, 261, 188-197.

45 C. Nick, S. Yadav, R. Joshi, C. Thielemann and J. J. Schneider, Beilstein J. Nanotechnol., 2014, 5, 1575-1579.

46 S. H. Ma, Q. Ye, X. W. Pei, D. Wang and F. Zhou, Adv. Mater. Interfaces, 2015, 2, 1500257.

47 A. J. Scardino, J. Guenther and R. de Nys, Biofouling, 2008, 24, 45-53. 